\title{
La parola denudata. La poesia come terreno di condivisione in Umberto Fiori
}

\author{
Matteo CURIONI \\ Università della Svizzera Italiana
}

\begin{abstract}
Il seguente articolo ha lo scopo di mettere in luce la riflessione sulla parola poetica nell'opera di Umberto Fiori, mediante una selezione di testi poetici tratti da diverse raccolte. A partire dalla frequente tematizzazione degli scambi verbali nei suoi lavori emergerà la forte tensione verso una parola che, cosciente dei propri limiti, riguadagni il suo profondo valore comunicativo per tornare ad essere segno di una possibile comunità umana.
\end{abstract}

Keywords: Umberto Fiori, parola, voce, esperienza, comunicazione

È possibile affermare senza timore che una delle spinte propulsive fondamentali della poesia di Umberto Fiori si trova nella costante tensione a mostrare l'evidenza del mondo reale, inteso come un dato di fatto tanto manifesto quanto imperscrutabile.

Lo strumento col quale tale scopo è perseguito, la parola, presenta tuttavia non pochi aspetti problematici. Essa si mostra sotto le vesti di una grande risorsa e contemporaneamente di uno strumento subdolo e manipolatore, capace di servire scopi opposti: se da un lato tendono alla chiarificazione, d'altro canto le parole possono essere impiegate per fissare le cangianti apparenze del mondo in formule fisse, nel tentativo di risolvere il problema della misteriosa evidenza della realtà e sostituirlo con una costruzione che ha il sapore dell'inganno.

Talvolta, nelle svariate discussioni che costellano le raccolte, si osservano personaggi intenti ad argomentare le proprie ragioni e convinzioni. Le parole, con il loro potere, sembrano dare sostanza a tutto ciò che sia enunciato con sufficiente sicurezza: dati, prove ed elementi vari si affollano nel discorso e permettono così a chi parla di imporre il proprio punto di vista:

\footnotetext{
Perché è così: quando ti chiama quello

che mette in ordine il mondo,

quello che tiene insieme

persone e cose, quello che costringe

a darsi ragione o torto

- non si scappa, tocca dar retta,

tocca rispondere all'appello. ${ }^{\mathrm{I}}$
}

I Convinto, da Chiarimenti, in Umberto Fiori, Poesie, Milano, Mondadori, 20I4, p. II3. 
Non è necessariamente espressione di malvagità o pura volontà di sopruso, ma piuttosto della forte esigenza di porre ogni cosa all'interno di un sistema che vuole essere il più possibile coerente e completo, espressione della volontà di razionalizzazione e comprensione globale del mondo. Lungo questa via si giunge irrimediabilmente a creare una distanza tra le parole e le cose, la cui causa è di fatto una disfunzione del meccanismo di referenzialità. Nel tentativo di imporre un certo ordine all'esistente, il locutore opera una cristallizzazione dei significati, riflesso di una cristallizzazione dell'esistente in forme predeterminate e immutabili, il che di fatto implica stabilire un diaframma tra la materia reale e la materia verbale censurando gli aspetti problematici e concentrandosi piuttosto sulla coerenza del discorso: «niente sostiene niente. Le parole / sono solo parole». ${ }^{2}$

Il disagio dell'io lirico si fa tangibile: la parola come gli è nota fino a quel momento appare inadatta a descrivere ciò che ha davanti agli occhi, al punto tale da fargli desiderare la fine di ogni discorso, per lasciare così spazio solo alla realtà:

La gente, da capo a piedi - basta guardarla:

è un bel mistero. Eppure quando parla

si riempie sempre la bocca

di spiegazioni.

In mezzo a tutti questi sapienti, uno

è solo come la salma

in mezzo alla camera ardente.

Non sta a sentire più, guarda via.

Guarda in un altro posto,

fuori, dove finiscono i discorsi

e i pianti, e le preghiere.

Là in fondo trova la ghiaia, il chiosco

e i platani e il fumo, splendenti

di calma e di compagnia. ${ }^{3}$

Questa parola non svela ma nasconde, piegando l'esistente in schemi forzati che tuttavia falliscono miseramente nel raggiungere il loro scopo; le conversazioni diventano scontri nei quali i partecipanti perdono di vista l'intento dello scambio verbale, lasciando una sconsolata amarezza a ricolmare gli animi, Un peso:

2 Fiori, Le parole, da Chiarimenti, cit., p. II6.

3 Fiori, Salma, da Chiarimenti, cit., p. 93. 


\author{
A ripensare agli argomenti \\ urlati sulla faccia, alle frasi \\ troncate subito a metà \\ oppure - peggio - lasciate cadere, \\ rimane come un peso. Anche stasera \\ ognuno ha detto la sua \\ senza che poi nessuno, \\ alla fine, \\ riuscisse a chiarire niente. ${ }^{4}$
}

La coerenza e la totale esaustività sono le chimere che l'uomo cerca in ogni discorso: insegue questo miraggio nel desiderio profondo di dare un senso a tutto ciò che hanno davanti, «come se con una frase / si potesse davvero dare e togliere, / legare e sciogliere e mettere bene in chiaro / tutto, una volta per sempre». ${ }^{5}$ Tale ossessione riporta dunque tutto il problema sullo sguardo e sulla relazione col mondo: la costruzione di discorsi e argomenti conduce alla perdita dell'esperienza al livello più basilare, portando i soggetti a vivere nella virtualità dei loro mondi fatti di pensiero; sistemi fittizi, mondi possibili ma non reali.

Occorre allora evitare le parole, riportare tutto a una esperienza che non sia dicibile, pena la sua irrimediabile riduzione? Una simile paralisi non è né può essere il traguardo auspicabile, ma una sconfitta radicale: il problema delle parole è la loro scissione dall'esperienza, non il fatto che esistano. Prova ne sia il fatto che, pur percependone la dolorosa inefficacia, l'io lirico non smette di riporvi la propria fiducia:
Ma sulla strada di casa, libero, ancora scosso per la discussione, ti commuove, a pensarci, ogni volta vedere quanta fede hanno nelle parole. ${ }^{6}$

La soluzione di questo scacco nella poesia di Fiori passa allora attraverso la riscoperta della struttura imprescindibilmente relazionale della parola, che rende possibile vincerne l'aspetto «finzionale». Come afferma il linguista Andrea Moro:

Una scienza che non ci dice niente di noi è inutile [...]. Se poi quella scienza studia il linguaggio, è impossibile che non ci dica qualcosa di noi - a meno di

4 Fiori, Un peso, da Chiarimenti, cit., p. II4.

5 Fiori, Le parole, cit., p. II5.

6 Ibidem. 
non volerlo - perché il linguaggio sta solo in noi, come i teoremi e le sinfonie; fuori ci sono oggetti, movimento e luce. Le costellazioni e le sinfonie ci sono solo perché ci siamo noi a guardare e ascoltare. Così per le frasi: quando le studiamo, infatti, ci troviamo, in un certo senso, nella situazione di chi studia la luce. Noi non vediamo la luce, vediamo solo gli effetti che ha sugli oggetti. Sappiamo della sua esistenza solo perché viene in parte riflessa da quello che incontra nel suo cammino, rendendo visibile ciò che altrimenti non lo sarebbe. Così un nulla, illuminato da un altro nulla, diventa per noi qualcosa. Allo stesso modo funzionano le frasi e le parole: non hanno contenuto in sé, ma se incontrano qualcuno che le ascolta diventano qualcosa. Diventano parte del dato. ${ }^{7}$

Affinché la parola torni ad essere un dato è necessario ritrovare l'aspetto relazionale, ovvero tornare a porre attenzione al destinatario, e sono proprio i momenti di incomprensione verbale ad avviare una tale riscoperta:

Quando mi sono accorto che era convinto

- in buona fede - di essere d'accordo

con me (anzi, voleva farmi capire meglio

quel che pensavo), e invece

aveva inteso male, male, proprio

una cosa per l'altra,

quando ho visto che era inutile

ormai spiegarsi ancora,

ho avuto un tuffo:

come quando di colpo va via la luce

e nella stanza le voci sono più chiare.

Due, tre frasi sospese,

qualche mezzo sospiro - poi nessuno

è più riuscito a parlare.

Lontano, in fondo al buio dell'orecchio,

la linea c'era: si sentivano

i cavi scoppiettare.

Con l'apparecchio in mano,

da una parte e dall'altra noi stavamo

curvi sopra l'equivoco

come sopra le fiamme di un focolare. ${ }^{8}$

7 Andrea Moro, Parlo dunque sono, Milano, Adelphi, 2013, p. I2.

8 Fiori, Telefonata, da Chiarimenti, cit., pp. II3-II4. 
La situazione è quella di una comunicazione imperfetta, una persuasione che non ha modo di attuarsi a dispetto delle migliori intenzioni: dal silenzio imbarazzato le voci si stagliano ancora più chiaramente nell'orecchio, imponendosi nella loro presenza che tuttavia si rivela ancora inefficace. Una condizione di privazione sensoriale conduce a un'esperienza più profonda $\mathrm{e}$ radicale, in cui tutte le possibili distrazioni e sovrastrutture sono cancellate; è il chiaro significato dell'immagine del black-out, nella quale la situazione insolita e disagevole diviene occasione privilegiata - tratto comune a tutti gli imprevisti che avvengono nel mondo poetico di Fiori. Quando le frasi sono penosamente menomate e sospese, si riconosce ciò che la fluidità di un discorso compiuto nasconde:
Si capiscono bene
questi discorsi; scorrono
sicuri, trovano un tono.
Arrivano, le parole,
da sole, come dette da qualcuno
qualche altra volta. ${ }^{9}$

Le parole sono un focolare, un luogo di ritrovo e di condivisione di esperienze; nel momento in cui dimentica di essere per l'altro il discorso esclude l'aspetto della reale comunicazione, pur essendo tecnicamente migliore. L'incomprensione che, in Telefonata come altrove, inspiegabilmente si manifesta anche a partire dalle premesse migliori, pone il parlante inevitabilmente di fronte all'assurdo della non comprensione e allo stesso tempo mostra la via da percorrere: il riconoscimento di una comunità, il tentativo di avvicinamento.

Le parole sono radicalmente e irrimediabilmente imperfette, se anche nella forma più precisa non sono in grado di penetrare e descrivere con esauriente efficacia quel buio che si trova in ogni uomo e al fondo del suo rapporto con la realtà. Dunque parlare separa, eppure paradossalmente la parola è anche l'unico mezzo capace di esprimere la reale comunione tra gli esseri umani, così come quella esistente tra loro e la realtà stessa. Come afferma Calvino nelle sue Lezioni americane:

Siamo sempre alla caccia di qualcosa di nascosto o di solo potenziale o ipotetico [...] la parola collega la traccia visibile alla cosa invisibile, alla cosa assente, alla cosa desiderata o temuta, come un fragile ponte gettato sul vuoto. ${ }^{10}$

9 Fiori, Lingua, da Esempi, in Fiori, Poesie, cit., p. 63.

Io Italo Calvino, Lezioni americane, Milano, Mondadori, 1993, p. 76. 
La soluzione non è allora un silenzio rassegnato all'impotenza, ma la considerazione onesta dei limiti e delle perdite, che semmai diventano un punto di partenza per recuperare ciò che si è perduto, ovvero «una comunità che si ritrova, senza strategie di sovranità o annessione»:"

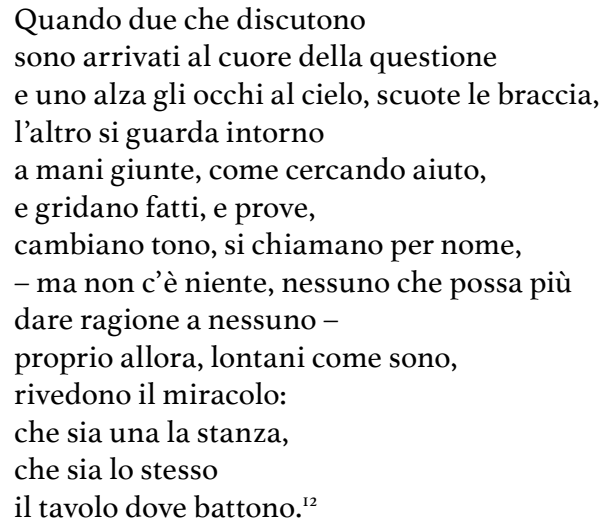

La parola mantiene anche la sua capacità di «dire» la realtà, perché il legame che intrattiene con essa è ancora vivo; il fatto che il linguaggio assuma una determinata forma riflette insomma la capacità umana di leggere il reale e riconoscerne le strutture, che poi in esso stesso si rispecchiano. La relazionalità inalienabile del linguaggio si esplicita dunque già al livello della sua stessa conformazione, che garantisce la possibilità di incontro e dialogo tra gli esseri umani. Nella poetica di Fiori, fatta di progressive riscoperte dei fondamentali dell'esperienza umana, la lingua ricopre un ruolo fondamentale perché rappresenta uno dei canali privilegiati con cui l'uomo può riscoprire la sua vocazione comunitaria sopita.

Esiste dunque una compenetrazione profonda tra esistente e parola: se per Ponge «le choses sono impastate di parola, in maniera irreversibile», ${ }^{13}$ in Fiori il linguaggio vive una medesima condizione, sebbene non sia concentrato sulle cose, quanto piuttosto sulla sua capacità di fondare un rapporto nonostante i suoi inevitabili limiti. Quello della limitatezza del codice linguistico è un tema cardine nelle poesie di Fiori, in cui l'io lirico si meraviglia del fatto che i suoi simili si ostinino a riporre fiducia nelle parole, nonostante tutta la loro dimostrata insufficienza:

II Andrea Afribo, Perdere tutte le bravure, in Fiori, Poesie, cit., p. XVII.

I2 Fiori, Altra discussione, da Esempi, cit., p. 52.

I3 Jacqueline Risset, De varietate rerum, o l'allegria materialista, in Francis Ponge, Il partito preso delle cose, Torino, Einaudi, 1979, p. IX. 
Vedi? Parlare ci separa. Eppure nemmeno nella stretta di mano più calda, occhi negli occhi, nemmeno abbracciati, persi nel bacio più profondo saremo mai vicini come siamo nelle parole. ${ }^{14}$

Con Ponge, si potrebbe chiosare questo inestricabile rapporto attraverso la sua celebre Il mollusco:

Il mollusco è dotato di un'energia potente nel rinchiudersi. A dire il vero non è altro che un muscolo, un ganghero, un blount e la sua porta.

Il blount ha prodotto la porta. Due porte leggermente concave formano tutta la sua dimora.

Prima e ultima dimora. Vi abita fin dopo la morte.

Niente da fare per estrarlo vivo.

Così, e con forza eguale, la più piccola cellula del corpo dell'uomo è attaccata alla parola, e viceversa. ${ }^{15}$

La riuscita dello scambio comunicativo non è affatto garantita: ciononostante, può sempre verificarsi il miracolo della comprensione, che consente alle parole di essere il più alto luogo di comunione umana.

L'aria vibra, noi ci capiamo:

è questo l'imbarazzo che ci tiene,

ci stringe insieme.

A volte due che si fermano appena

per un saluto, un attimo,

quasi li schiaccia. E se non parlano rimane, e cresce, e sembra l'unica cosa chiara. ${ }^{16}$

Chi ascolta non può fare a meno di comprendere le parole che il suo interlocutore gli rivolge: nel loro stesso materiale verbale si trova intessuta la finalità comunicativa, baluardo incrollabile perfino nei momenti in cui domina l'imbarazzo dell'incapacità o dell'incompletezza. Questo è il miracolo, un inalienabile terreno comune che l'uomo non potrà mai sradicare da sé. Ecco il motivo della fiducia nelle parole: se comunicazione può avvenire, il luogo privilegiato non può che essere questo.

I4 Fiori, Spiegarsi, da Esempi, cit., p. 42.

I5 Ponge, Il partito preso delle cose, cit., p. 39.

I6 Fiori, Spiegarsi, da Esempi, cit., p. 42. 
Rimane però in primo piano il problema dell'oggetto dello scambio: per i personaggi di Fiori infatti una comunicazione completa ed esauriente rimane un traguardo lontanissimo; del resto, se anche si riuscisse a raggiungere la chiarezza più estrema, l'io lirico non saprebbe come metterla a frutto:

\author{
E anche se un giorno il relatore \\ riuscisse a stringere meravigliosamente \\ tema e pubblico in pugno \\ - nel suo pugno peloso di scimmione - \\ e ci portasse su, \\ fino al cielo stellato, tutti insieme, \\ e lassù trattenessimo insieme il fiato \\ come prima di un tuffo, e l'interesse \\ tenesse il tempo sospeso \\ finché, oltre le ultime poltrone, \\ si vedesse brillare la promessa \\ di avere finalmente le cose chiare, \\ comunque si dovrebbe ritornare \\ a casa, perché è già tardi, \\ si è fatta l'ora di cena. ${ }^{17}$
}

Il problema non è tanto la finezza delle considerazioni, quanto piuttosto la rilevanza che le parole assumono nell'esperienza. Si comprende così perché i momenti più significativi sono quelli in cui queste acquisiscono una sostanza quasi materica, un'evidenza in qualche modo tangibile, come quella realtà che si incaricano di rappresentare ma che rimane inesauribile.

Quando un tram carico di gente

ti lascia a un incrocio, e sei solo

sul piazzale, davanti a un casamento

che in piena luce sta lì

piantato, chiaro, chiuso come un monte,

ti sembra di capire bene,

eppure non sai rispondere.

Ma poi a volte dentro

- giù, giù, sul fondo,

dove tutto il fiato è finito

e niente si lascia dire - viene una frase

e senti che sta già in piedi, che è viva,

che è vera come un naso, come una mano. ${ }^{18}$

I7 Fiori, Conferenze, da Chiarimenti, cit., p. I20.

18 Fiori, Frase, ibid., p.33. 
La reale pregnanza dei discorsi sta insomma nel rapporto che la parola instaura, e nel suo continuo rimandarvi: ciò che conta è la reale pertinenza che lega le parole ai soggetti e alla realtà. A ben guardare quindi il vero problema non riguarda unilateralmente il polo dell'alterità, intesa sia come realtà dicibile che come interlocutore; l'elemento cruciale sembra essere soprattutto l'enunciatore del discorso. Si tratta insomma di recuperare entrambe le parti che rendono "dato" i discorsi e le frasi, ovvero l'enunciatore e il destinatario, attraverso l'azione di un soggetto che sia capace di mettere da parte l'ambizione di un discorso perfetto ed essere invece presente con tutto sé stesso in ciò che tenta di affermare. È qui che diventa fondamentale quel «perdere le bravure», lasciare da parte tutte le tecniche per tentare di esprimere e di realizzare quel desiderio di chiarimento implicito - ma sempre percepibile, anche se a livello involontario - ad ogni dialogo.

È come se in quei discorsi

la gloria che rimane sempre nascosta potesse prima o poi venir fuori.

Ti stanno in testa ormai, queste serate, come ai bambini

la notte di Natale.

Sempre un dunque ti aspetti da quelle quattro chiacchere, una stretta finale, un chiarimento.

Invece, niente: a parte quando si ride nessuno è poi lì dov'è, nessuno parla - $\mathrm{o}$ ascolta - veramente.

(E certo è anche bello, e strano, e grande anche, a pensarci, tenere a bada così alla buona, con poco, la verità.)

Ma a volte si ragiona, intorno al tavolo, si fa sul serio: comincia una discussione.

\section{$[\ldots]$}

Non che con queste mezze frasi prepari un discorso - invece - compiuto.

Non cerca più un'altra voce, più vera:

trova la sua già qui, 
tutta intera,

e prova a parlare.

Prova cos'è parlare, avere una voce, cos'è essere poveri.

\section{$[\ldots]$}

Lo vedi da dove viene la voce, e che cos'ha uno che canta.

Ha che le ha perse tutte, le bravure.

Le cose non le sa più dire bene:

soltanto così, soltanto perfettamente ${ }^{19}$.

Non è egoismo o prevaricazione sugli altri, questo "canto" è piuttosto la totale esposizione di sé stesso, del proprio reale pensiero, corredato da ogni possibile limite o contraddizione e aperto così a tutte le possibili critiche: «solo chi ha parlato veramente / può veramente essere frainteso». ${ }^{20} \mathrm{E}$ in effetti il primo passo per un vero dialogo, manifestare un pensiero che scaturisca dai nodi più cruciali dell'esperienza del soggetto, quel luogo che ciascuno condivide con tutti gli altri suoi simili, in modo tale che «con questa musica rauca, velata, / la cosa che è nascosta dentro il mondo / rischia la vita»:21 non la risposta dunque, ma la tematizzazione onesta delle domande. Tutte le strategie vengono messe da parte e nel discorso può trovare spazio solo ciò che più urge nell'animo in quel preciso momento: ciò che sembra egoismo è invece la condizione affinché le parole possano ritrovare la loro natura, svelando la realtà anziché coprirla. Trovare questa dimensione più autentica significa però riconoscerne anche il limite intrinseco: esse sono drammaticamente insufficienti e nella poesia l'oratore è costretto ad aiutarsi con i gesti. Paradossalmente, «meno gli stanno in piedi gli argomenti, / più sente in bocca il discorso / dargli ragione»: «sono pronte, le parole. / Gli stanno in faccia / e non dicono niente ${ }^{22}$.

Il vero discorso non può essere in alcun modo esauriente perché inevitabilmente raggiunge dei misteri che non hanno una soluzione univoca ed evidente: le parole, nel loro impiego più proprio e adatto, ricoprono una funzione descrittiva della realtà; in un modo simile a quanto affermava

I9 Fiori, Il discorso e la voce, da Chiarimenti, cit., pp. 75-8I.

20 Fiori, Un peso, da Chiarimenti, cit., p. II5.

2I Fiori, Il discorso e la voce, cit., p. 8I.

22 Ibidem, p. 77. 
Wittgenstein, esse non devono cedere alla tentazione di imporsi come creatrici di significato, ma limitarsi a registrare ogni scacco e ogni rinascita, sforzandosi di trovare il modo più appropriato per comunicare il reale. La poesia di Fiori abbraccia uno stile scarno per mostrare quanta profondità si trovi nell'esperienza più quotidiana: da ciò si origina una parola ostinata, occasione di rinascita dopo troppi momenti passati a sprecare fiato inutilmente, che instancabilmente cerca la realtà sotto il velo delle ideologie e delle riduzioni. Finalmente il soggetto ha l'occasione di esprimere quanto ha di più caro, per quanto vaga possa esserne la sua percezione, teso a un momento in cui la promessa che implicitamente aleggia su ogni incontro possa raggiungere un almeno parziale compimento.

Punto di svolta è insomma trovare il coraggio di esprimere i nodi, i desideri, le paure e le domande che costituiscono l'intimo tessuto dell'esistenza umana. Perdere le bravure è anche questo: lasciare che la poesia diventi «solo voce», avendo il coraggio di esporsi e, contemporaneamente, di esporre e riflettere. È forse questa allora la definizione della poesia di Fiori, un canto, un verso, una voce - la propria. Il poeta «di voce» cerca una parola che è già sua, "perché è lui ad appartenere a quella parola»²3: «caro e naturale possesso, una scheggia e un suono della sua vita», ${ }^{24}$ denudata perché non è trattata come una materia da modellare, irrelata e separata dal sé: al contrario essa è per figlia della mancanza, di cui si trova ad essere espressione.

Non si tratta dunque di fondare la legittimità della parola poetica - 0 , per esteso, della propria parola in generale - su quelle di un soggetto sociale o collettivo, piuttosto «il percorso è inverso: è la parola davvero comune, quando si fa riconoscere, a indicare il cammino verso una comunità di cui reca il segno, la traccia, la voce». ${ }^{25}$ Insomma, la parola si fa davvero comunitaria nel momento in cui diviene portatrice delle più reali tensioni del singolo: «la condizione del poeta è quella di un animale che ha dimenticato il proprio verso. [...] Questo è la voce: raglio, fischio, muggito che scopre cosa siamo e ci scopre, ci mette allo scoperto, ci espone». ${ }^{26}$ D'altronde, forse è proprio questo legame quasi organico a permettere di avvicinarsi al mistero dell'insondabilità del proprio sé. Il fare del poeta è più un ascolto, nel quale troverà la parola che impone «la sua assoluta familiarità», scaturita da una povertà, «da un radicale bisogno di parole» - condizione che forse può essere avvicinata alle considerazioni di Barthes circa lo «stile», inteso come

23 Fiori, La poesia è un fischio, Milano, Marcos y Marcos, 2003, p. 2I.

24 Rainer Maria Rilke, Lettere a un giovane poeta, traduzione di Marina Bistolfi, Milano, Mondadori, 20I3, p. 40.

25 Fiori, La poesia è un fischio, cit., p. 23.

26 Ibidem, p. 24. 
un fatto biologico, «quasi che, in una specie di crescita floreale, lo stile fosse solo il termine di una metamorfosi, cieca e ostinata, la parte di un infralinguaggio che si elabora al limite tra la carne e il mondo». ${ }^{27}$

La trattazione della voce in Fiori costituisce evidentemente una presa di distanza da qualunque neutralizzazione della voce poetica; come egli stesso afferma in La poesia è un fischio, superare una simile posizione implica anche superare una poesia in cui «l'oggetto estetico, il testo, finisce per prevalere sul soggetto del sentire e dell'esprimere, fino a imporgli un nuovo ritegno, una nuova austerità», ${ }^{28}$ quella «disumanizzazione dell'arte» di cui parla Ortega y Gasset.

La parola poetica secondo Fiori deve dunque essere sempre cosciente della propria origine inestricabilmente legata all'individualità - e qui si colloca la pertinenza dello stile nella formulazione «biologica» di Barthes - che però non si considera fuori dall'ordinario, perché fondamentalmente esprime tratti a cui chiunque può relazionarsi. Il «canto» allora non è semplice arbitrio personale o ostentazione dell'ego perché il poeta è pienamente responsabile delle sue parole e non pretende di esprimere individualità o esperienze fuori dall'ordinario: per questo l'io lirico di Fiori è sempre un chiunque. "Canto è, in una parola, etica e poesia» ${ }^{29}$ - non intesa certo come norma morale soggetta ai tempi e alle culture: il poeta che «canta» si rende disponibile a un ascolto chiedendo parallelamente di essere ascoltato. Egli non fa un discorso perfetto nel senso di culmine di una scala di valori, conclude l'autore nel suo La poesia è un fischio: è perfetto in senso assoluto, ovvero è compiuto. La poesia mostra la nostra condizione, il nostro stare al mondo e in questo senso essa è etica, tesa ad affermare innanzitutto che quanto diciamo è e deve ambire ad essere rilevante - non necessariamente giusto. ${ }^{30}$ «Ecco l'insegnamento della poesia, alto e terribile».31

È questa tensione che permette all'opera di Fiori di essere al contempo inesplicabilmente legata all'individualità e all'anonimato, insomma al «rimettersi sulle tracce della voce come singolare ma impersonale fondamento della parola poetica, sua unità, sua vera forma e dunque suo vero problema».32 La voce è portatrice di ciò che siamo, ci manifesta agli altri, almeno in parte: ancora una volta infatti non esiste la comunicazione completa e perfetta: il mistero rimane presente, la pretesa di una totale chiarezza inevitabilmente disattesa: "parlare / è sempre troppo / e non è mai abbastan-

27 Roland Barthes, Il grado zero della scrittura, Torino, Einaudi, I982, pp. Io-II.

28 Fiori, La poesia è un fischio, cit. p. 25.

29 Ibidem, p. 34.

30 Parafraso da Fiori, La poesia è un fischio, cit., p. 38.

3I Ibidem.

32 Ibidem, p. 27. 
za».33 La poesia dunque mostra, offre e interroga, forte di una componente ostensiva che si propone come caratteristica primaria della parola, luogo di ritrovo e occasione di incontro.

mt.curioni@gmail.com

33 Fiori, Chiarimenti, da Chiarimenti, cit., p. Ioo. 